\title{
Double spin asymmetry measurement from SANE-HMS data at Jefferson Lab
}

\section{Hoyoung KANG*†}

Seoul National University, Seoul 151-742, South Korea

E-mail: achim50esnu.ac.kr

In Hall $\mathrm{C}$ at the Thomas Jefferson National Laboratory, the Spin Asymmetries of the Nucleon Experiment (SANE, E07-003) measured the spin asymmetries of the proton, using a polarized electron beam and a polarized ammonia target. By rotation of superconducting magnet about the target, parallel and near-perpendicular double spin asymmetries were measured at beam energies of 4.7 and $5.9 \mathrm{GeV}$. The spin structure functions $g_{1}$ and $g_{2}$ can be extracted from these asymmetries. The primary experiment used the Big Electron Telescope Array (BETA) which covered a large solid angle (about 200msr). Simultaneously, the High Momentum Spectrometer (HMS) detected electrons scattered. Double spin asymmetry from HMS data can improve the integral of $g_{2}$ and the $Q^{2}$ dependency of $A_{1}$ and $A_{2}$ at resonance region. We show the current progress of the analysis and preliminary spin asymmetries from HMS data.

XXI International Workshop on Deep-Inelastic Scattering and Related Subjects 22-26 April, 2013

Marseilles, France

\footnotetext{
* Speaker.

${ }^{\dagger}$ For SANE Collaboration
} 


\section{Introduction}

The Spin Asymmetries of the Nucleon Experiment (SANE, TJNAF E07-003) was a measurement of the spin asymmetries of the proton. It was done in Hall $\mathrm{C}$ at Thomas Jefferson National Laboratory, or Jefferson Lab, from January to March 2009. Using a polarized electron beam and a polarized ammonia target, it produced data for parallel and near-perpendicular asymmetries. The world data of proton spin asymmetries, especially perpendicular asymmetry $A_{\perp}$, still lacks big kinematic region, except some data points[1][2][3]. SANE will cover broad range of high Bjorken $x$, $0.3<x<0.8$, and four-momentum transfer $2.5 \mathrm{GeV}^{2}<Q^{2}<6.5 \mathrm{GeV}^{2}$ [4]. SANE used two detector complexes, Big Electron Telescope Array (BETA) and High Momentum Spectrometer (HMS) for this purpose. BETA covered a large solid angle of 200msr , and served as a main detector. HMS, a built-in spectrometer of Hall C, collected complementary data.

Physical motivation of the experiment is to improve our understanding about proton spin structure functions. It is concentrated more on transverse spin structure, which is a structure function $g_{2}$. Having both parallel and perpendicular asymmetries, two spin structure functions, $g_{1}$ and $g_{2}$, can be extracted directly, without relying on Wandzura-Wilczek relation [5], for spin asymmetries are linear combination of spin structure functions.

Even though BETA was the main detector, HMS collected also good amount of data. In the previous experiment of Resonance Spin Structure, only HMS was used to produce $g_{1}$ and $g_{2}$ [3]. So HMS data was independently analysed and showed good agreement with other experiments. HMS detected electrons scattered to beam right. Although BETA covers larger kinematic region, the HMS, by varying the central angle and momentum, collected complementary data at four-momentum transfer $Q^{2}$ of $0.8,1.3$, and $1.9 \mathrm{GeV}^{2}$, from the resonance region to the region of invariant mass $W=2.3 \mathrm{GeV}$. These regions are the extended range of previous RSS experiment, allowing better determination of the integral of $g_{2}$ and the $Q^{2}$ dependency of $A_{1}$ and $A_{2}$ at resonance region.

\section{Dilution Factor}

Figure 1 is the schematic view of the experiment. As an inclusive double spin asymmetry measurement, it used polarized electron beam of 4.7 and $5.9 \mathrm{GeV}$ energies and polarized solid ammonia target. Average beam polarization was about $73 \%$ and target proton 67\%. BETA and HMS detected electrons.

Granular type of ammonia makes packing fraction issue. Target cell is not completely filled with solid ammonia. So, actual amount of material should be derived from data. Using Monte Carlo simulation based on empirical fit of inelastic cross section [6], unpolarized cross section assuming targets of 50\% and 60\% packing fraction can be compared with actual HMS data. It determines packing fraction and dilution factor. Technical details are the same as the method used in RSS experiment [7]. Experiment and MC result shows good enough agreement to determine it. SANE packing fractions are $56-62 \%$ with $4.5 \%$ point error.

\section{Asymmetry Results}

Successful determination of dilution factor and beam and target polarization finally made it 


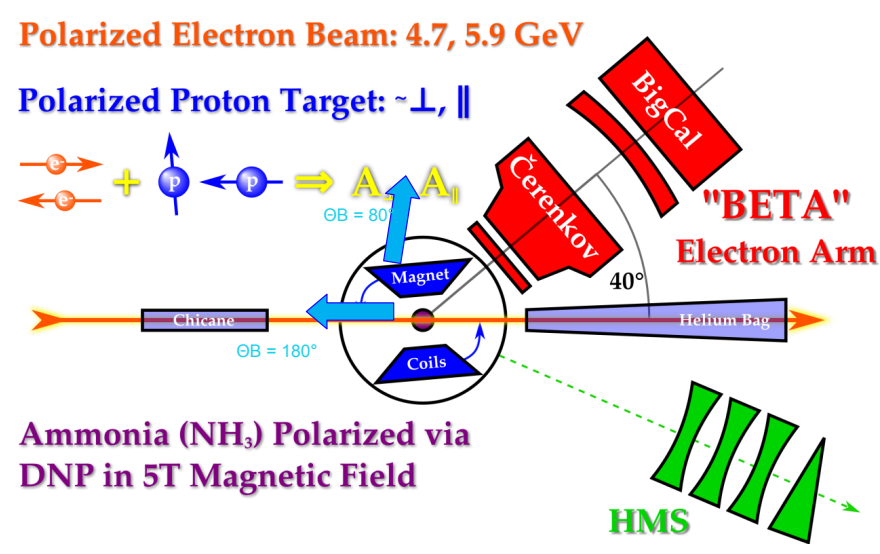

Figure 1: SANE layout : Polarized electron beam is scatted by polarized ammonia target. BETA and HMS detects electrons.

possible to get HMS asymmetries. HMS covers three major kinematic regions at four-momentum transfer $Q^{2}$ of $0.8,1.3$, and $1.9 \mathrm{GeV}^{2}$. These regions have both parallel and near-perpendicular asymmetry data, so can be used to determine physics asymmetry $A_{1}$ and $A_{2}$ directly. Kinematic calculation converts $A_{\|}$and $A_{\perp}$, that is actually $A_{80^{\circ}}$, into physics asymmetry. This calculation follows [8].

After dividing raw asymmetries by beam and target polarization and dilution factor, it also requires radiative correction. Radiative correction uses code based on POLRAD [9]. Now we have preliminary asymmetries radiative-corrected.

Preliminary asymmetries are consistent with previous experiments [3][10]. All the plots are $W$ spectra, where $W$ is the invariant mass of recoil system. Discrepancies in Figure 2 can be a sign of high dependency of resonance on $Q^{2}$. Figure 3 covers the deep inelastic scattering regions.

\section{Conclusion}

SANE-HMS data is expected to extend our understanding of $Q^{2}$ dependencies and integrals of $g_{2}$. HMS data produced packing fraction and dilution factor successfully. SANE analysis are in good progress, including preliminary HMS asymmetries. HMS asymmetries are consistent with previous experiment.

\section{Acknowledgements}

SANE was supported by the Department of Energy and various institutions. This work was sponsored by Q2C World Class University fund at Seoul National University.

\section{References}

[1] K. Abe et al. (E143 Collaboration), Phys. Rev. D58, 112003 (1998) 

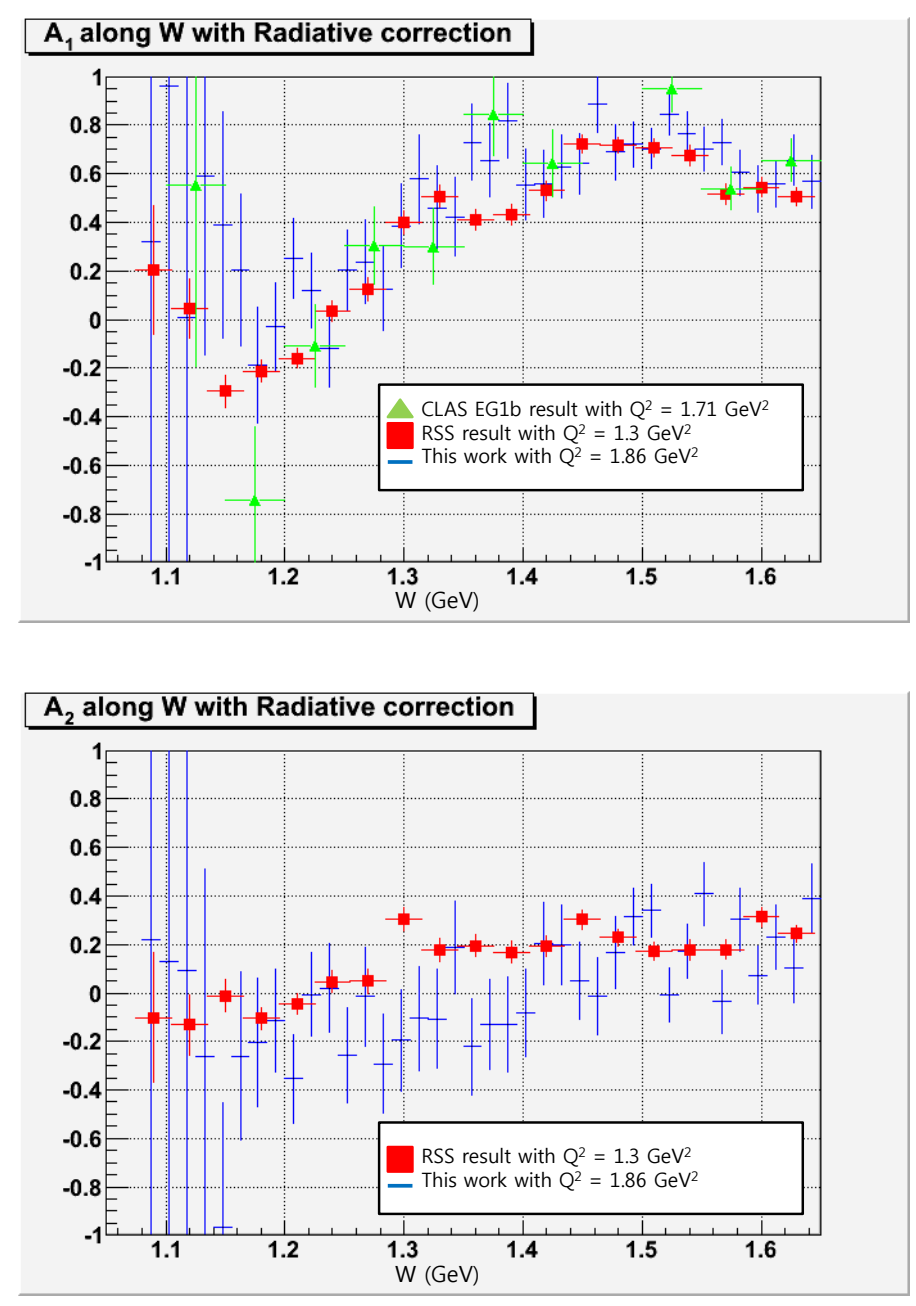

Figure 2: Preliminary $A_{1}$ and $A_{2}$ with $Q^{2}=1.86 G e V^{2}$ (Only statistical error included)

[2] P.L. Anthony et al. (E155 Collaboration), Phys. Lett. B458, 529 (1999)

[3] F.R. Wesselmann et al. (RSS Collaboration), Phys. Rev. Lett. 98, 132003 (2007)

[4] J-P. Chen, et al., Spin Asymmetries on the Nucleon Experiment, TJNAF PAC24 proposal (2003)

[5] S. Wandzura and F. Wilczek, Phys. Lett. 72B, 195 (1977)

[6] P.E. Bosted and M.E. Christy, Phys. Rev. C77, 065206 (2008)

[7] O.A. Rondon, The Packing Fraction and Dilution Factor in RSS, RSS technote 2005-03 (2006)

[8] M. Anselmino, et al., Phys. Rep. 261, 1 (1995)

[9] I. Akushevich, et al., Acta Phys. Polon. B28, 563 (1997)

[10] K.V. Dharmawardane, et al. (CLAS Collaboration), Phys. Lett. B641 11 (2006) 

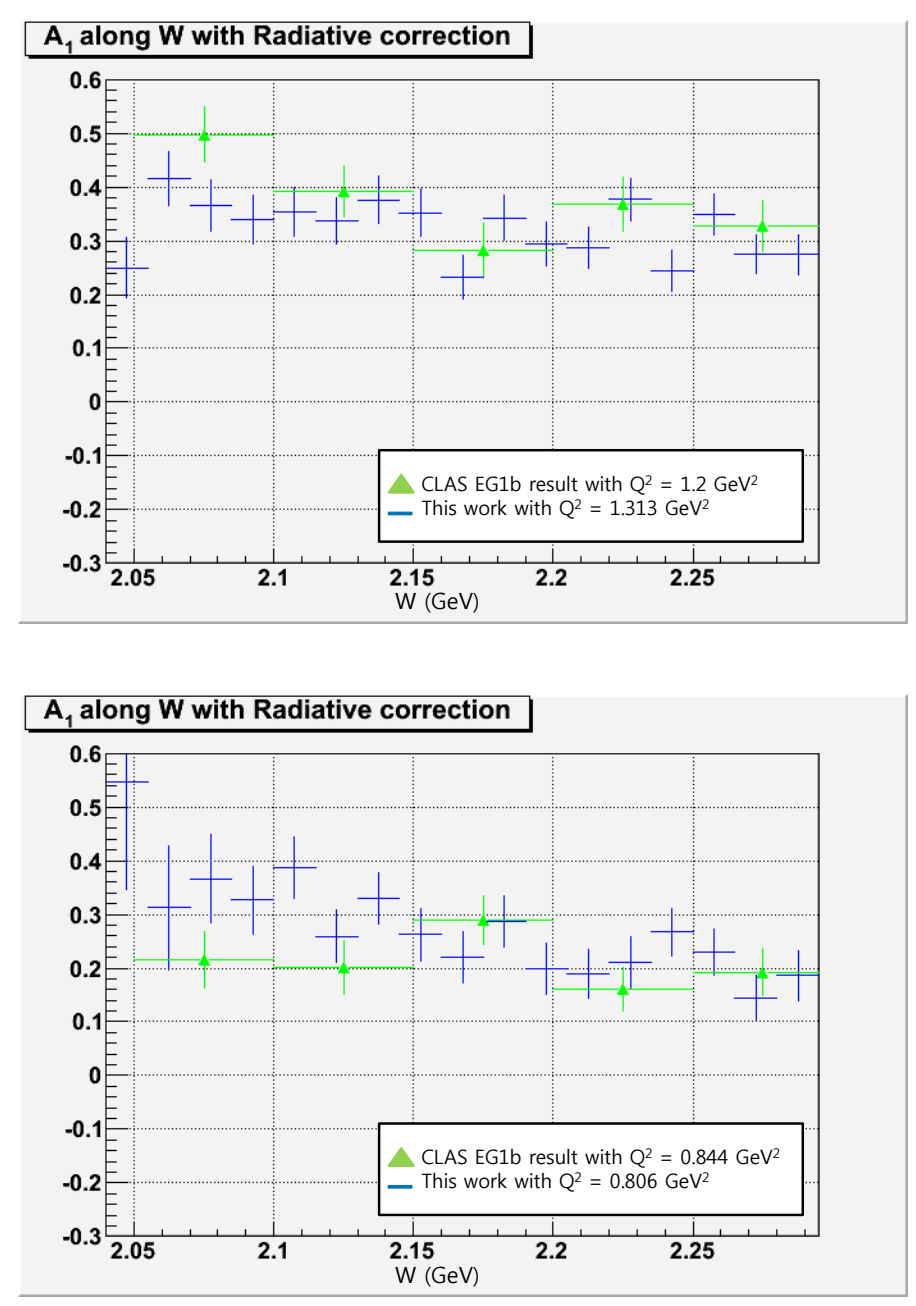

Figure 3: Preliminary $A_{1}$ with $Q^{2}=1.313 \mathrm{GeV}^{2}$ and $0.806 \mathrm{GeV}^{2}$ (Only statistical error included) 